\title{
Comparison of submucosal tunneling biopsy versus EUS-guided FNA for gastric subepithelial lesions: a prospective study with crossover design $\square$
}

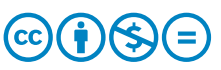

\author{
Authors \\ Hideki Kobara ${ }^{1}$, Hirohito Mori ${ }^{1}$, Naoki Nishimoto², Shintaro Fujihara', Noriko Nishiyama', Maki Ayaki ${ }^{1}$, Tatsuo \\ Yachida', Tae Matsunaga', Taiga Chiyo ${ }^{1}$, Nobuya Kobayashi ${ }^{1}$, Koji Fujita ${ }^{1}$, Kiyohito Kato ${ }^{1}$, Hideki Kamada ${ }^{1}$, Makoto \\ Oryu $^{1}$, Kunihiko Tsutsui ${ }^{1}$, Hisakazu Iwama ${ }^{3}$, Reiji Haba ${ }^{4}$, Tsutomu Masaki ${ }^{1}$
}

Institutions

1 Department of Gastroenterology and Neurology, Faculty of Medicine, Kagawa University, Kagawa, Japan

2 Department of Clinical Research Support Center, Faculty of Medicine, Kagawa University, Kagawa, Japan

3 Life Science Research Center, Faculty of Medicine, Kagawa University, Kagawa, Japan

4 Department of Diagnostic Pathology, Faculty of Medicine, Kagawa University, Kagawa, Japan

submitted 12.12 .2016

accepted after revision 22.5.2017

Bibliography

DOI https://doi.org/10.1055/s-0043-112497 |

Endoscopy International Open 2017; 05: E695-E705

(c) Georg Thieme Verlag KG Stuttgart · New York

ISSN 2364-3722

Corresponding author

Hideki Kobara, MD, PhD, Department of Gastroenterology and Neurology, Faculty of Medicine, Kagawa University,

1750-1 Ikenobe, Miki, Kita, Kagawa 761-0793, Japan

Fax: +81-87-891-2158

kobara@med.kagawa-u.ac.jp

\section{ABSTRACT}

Background and study aims Endoscopic ultrasoundguided fine needle aspiration (FNA) for gastrointestinal subepithelial lesions (SELs) has limited diagnostic accuracy due to technical problems and small lesion size. We pre- viously reported a novel submucosal tunneling biopsy (STB) technique for sampling SELs. This study aimed to evaluate the diagnostic ability and safety of STB compared to that of FNA for SELs.

Patients and methods The study was a non-randomized, prospective comparative study with crossover design in patients with endoluminal gastric SELs. Forty-three patients, including 29 cases with lesions $<2 \mathrm{~cm}$ were enrolled. A crossover design with 2 intervention stages (Group A: FNA followed by STB for 23 SELs, Group B: STB followed by FNA for 20 SELs) was implemented. The primary outcome was the diagnostic yield (DY). Secondary outcomes were technical success rate, procedure time, complication rate, and sample quality.

Results The DY of STB was significantly higher than that of FNA $(100 \%$ vs. $34.8 \% ; P<0.0001)$ in group $A$, including $100 \%$ in overall STB. The technical success rate of STB was significantly higher than that of FNA ( $100 \%$ vs. $56.5 \%$; $P=0.0006)$, whereas the median procedure time of STB was significantly longer than that of FNA (37 minutes vs. 18 minutes; $P<$ $0.0001)$. The median specimen area of STB samples was markedly larger than that of FNA samples $\left(5.54 \mathrm{~mm}^{2} \mathrm{vs}\right.$. $\left.0.69 \mathrm{~mm}^{2} ; P<0.001\right)$. No complications occurred in either method.

Conclusions STB had significantly superior diagnostic ability and a more adequate sample quality than FNA for endoluminal gastric SELs, indicating the suitability of STB for small SELs.

\section{Clinical trial registration: UMIN 000006754}

\section{Introduction}

Gastrointestinal subepithelial lesions (SELs) mainly involve mesenchymal tumors, such as gastrointestinal stromal tumors (GISTs), leiomyomas, and schwannomas, followed in frequency by heterotopic pancreas, cyst, lipoma, etc. [1]. GISTs, the most frequent type of SEL, have malignant potential and thus typically require surgical treatment [2]. Recently, minimally invasive local resection has been developed for intramural GISTs [3]. However, for indefinite SELs, management based on lesion size and tissue sampling methods must be further developed. 
Major guidelines offer similar recommendations for management of GISTs. The National Comprehensive Cancer Network (NCCN), European Society for Medical Oncology (ESMO), and Japanese guidelines state that lesions $\geq 2 \mathrm{~cm}$ could be excised and biopsied [4-7]. The NCCN guideline states that the 2-cm cutoff is rather arbitrary, although reasonable [4], and Canadian guidelines indicate that even GISTs $<1 \mathrm{~cm}$ could be resected because of the risk of metastasis [8]. Moreover, some authors have recently reported that small GISTs without highrisk features on endoscopic ultrasound (EUS) progressed rapidly or resulted in metastatic disease $[9,10]$. Thus, the management of small lesions $<2 \mathrm{~cm}$ remains controversial.

EUS is a key procedure in evaluation of gastrointestinal SELs. However, EUS morphologic features alone have limited specificity among the diverse subtypes of SELs [11]. Therefore, several tissue sampling methods have been proposed for diagnosis of SELs, with EUS-guided fine needle aspiration (FNA) emerging as a standard method. The diagnostic rate of FNA, including spindle cell neoplasms ("suspicious"), is moderately satisfactory (approximately $80 \%$ ) $[12,13]$. However, the immunohistological (IH) analysis required for a definitive final diagnosis revealed a limited diagnostic rate of FNA of approximately 50 $60 \%$ [13-15]. Thus, new techniques with a higher diagnostic yield are needed to acquire adequate specimens for $\mathrm{IH}$ analysis.

We previously developed a novel sampling method called submucosal tunneling biopsy (STB), which involves submucosal endoscopy with a mucosal flap (SEMF) [16], to obtain core biopsy specimens for growing endoluminal SELs $[17,18]$. The technical advantage is its use of a submucosal tunnel with SEMF, which makes it possible to visually identify the tumor itself, to acquire a core specimen of sufficient size for $\mathrm{IH}$ analysis, and to prevent delayed complications.

Hence, this prospective study aimed to compare the histologic diagnostic yield and safety of STB and EUS-FNA in patients with gastric SELs. We also evaluated the quality of histologic specimens obtained using these sampling methods.

\section{Patients and methods}

\section{Study population}

Between November 2011 and January 2016, 57 patients with gastric SELs were recruited and examined with routine EUS (high-frequency miniprobe, $20 \mathrm{MHz}$, UM-3R; Olympus Medical Systems, Tokyo, Japan) and abdominal computed tomography. The inclusion criterion was presence of a gastric SEL with primarily endoluminal growth. Then, patients meeting the following criteria were enrolled: physical status I-II, normal complete blood count, and normal prothrombin time. Exclusion criteria were as follows: age $<20$ years, obvious diagnosis of lipoma or cyst on EUS, lesion size $>5 \mathrm{~cm}$, for which the Japanese GIST guideline recommends surgical operation without preoperative histological diagnosis, and a lack of the patient's consent.

\section{Study design}

This study was a prospective, non-randomized, and comparative with crossover design conducted at a single academic medical center, Kagawa University Hospital, Japan. Patients were

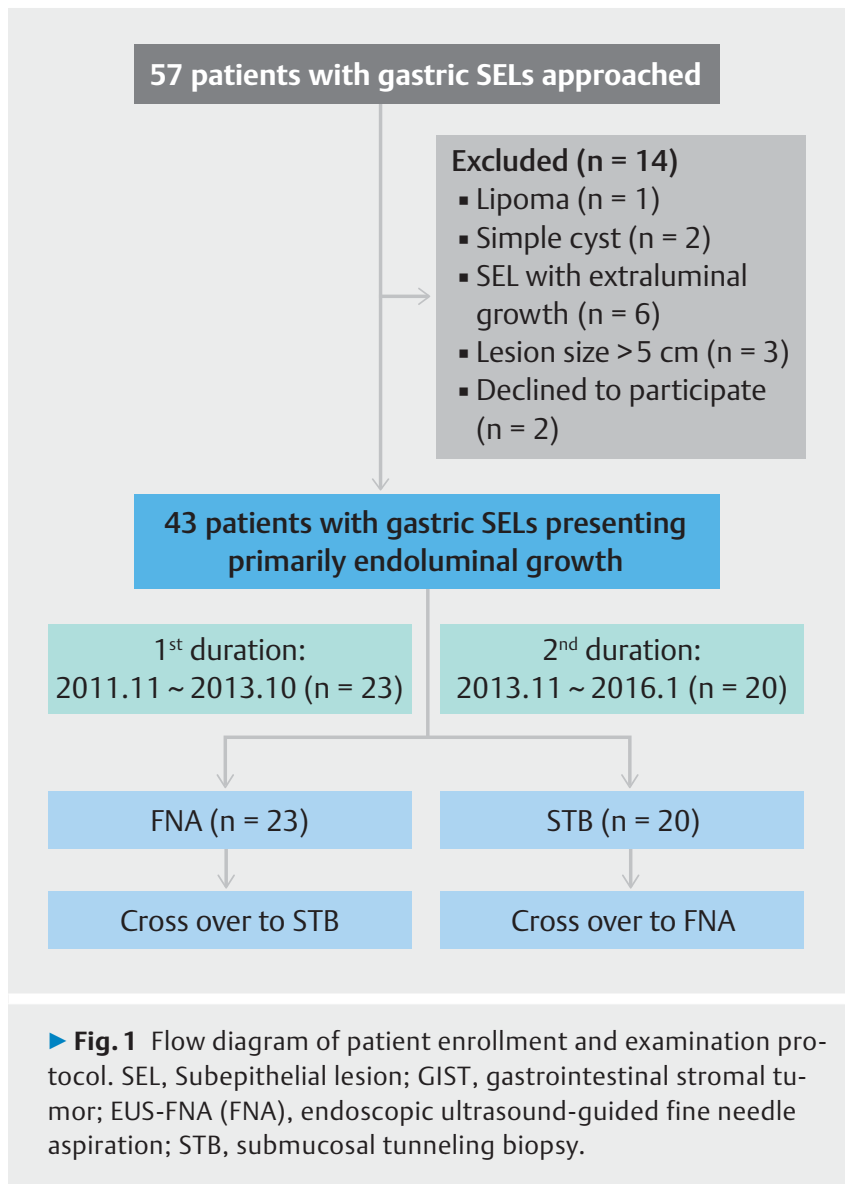

divided into two groups (Groups A and B), and a crossover study design with 2 intervention stages was implemented ( $>$ Fig. 1). The first intervention stage was performed between November 2011 and October 2013, and 23 patients scheduled to undergo FNA followed by STB were enrolled (Group A). The efficacy and safety of both FNA and STB were verified at the end of the first stage. The second intervention stage was between December 2013 and January 2016, and 20 patients scheduled to undergo STB followed by FNA were enrolled (Group B). In this study, the crossover design was applied to convert variations among individuals to the variation within individual, thus improving the estimated accuracy of the diagnostic yields. However, because there were few safety data on a novel STB, the study protocol considering the safety was incorporated referring to 2-stage design. With this design, an investigator would stop a clinical trial with monitoring the data either if unknown severe adverse events (AEs) associated with the experimental therapy happened with a frequency of approximately $15 \%$ or if there were sufficient evidence of efficacy to warrant phase III testing. Thus, after we verified the invasiveness and safety of STB at the Group A, we planned to proceed to Group B.

A 24-hour washout period was assigned to avoid carry-over of adverse events on day 3 between the procedures.

The current study was approved by the Clinical Ethics Committee of Kagawa University Hospital in accordance with the Declaration of Helsinki and was registered as University Hospi- 


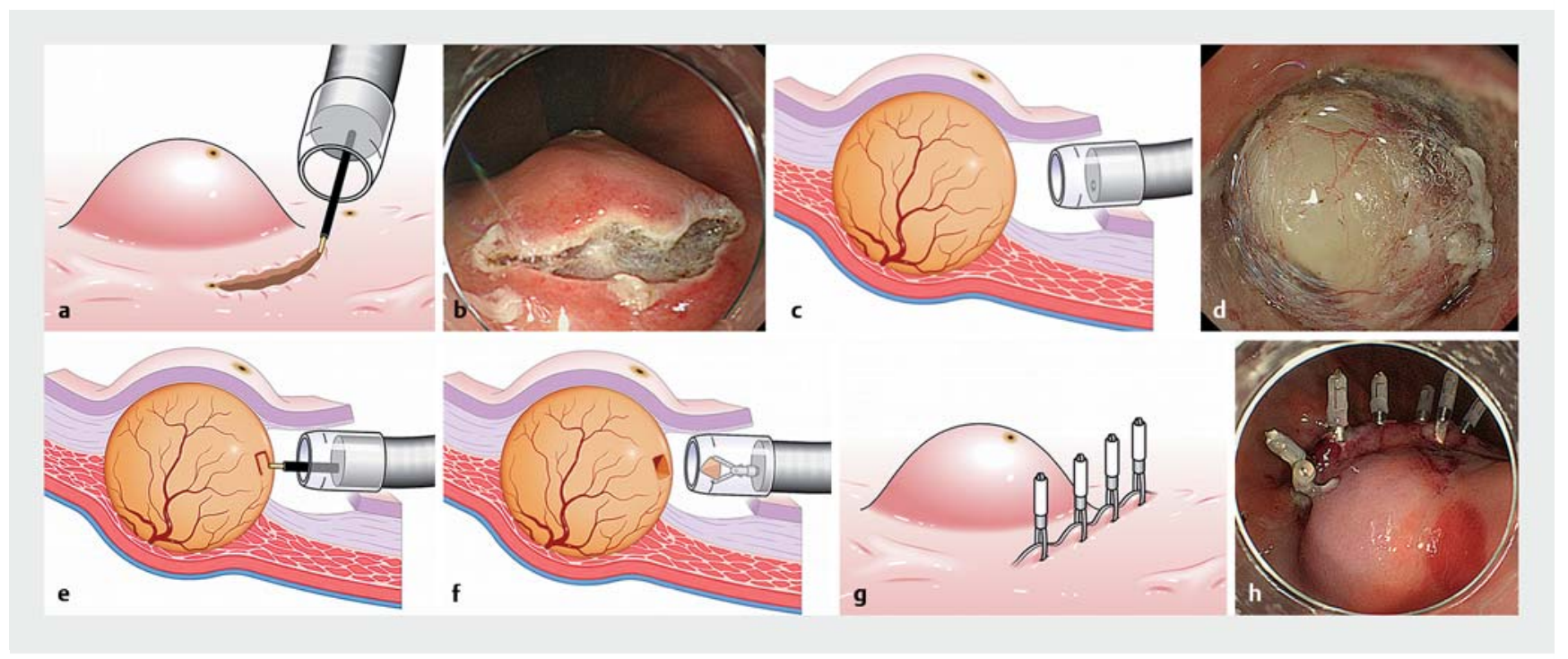

- Fig. 2 Submucosal tunneling biopsy (STB) procedure for subepithelial lesions. a Creation of the entry: A 10-mm opening flap is created by mucosal incision and submucosal dissection after marking two dots around the lesion at a margin of approximately $5 \mathrm{~mm}$, with one dot at the top of the lesion. b Endoscopic image showing a 10-mm opening flap. c Submucosal endoscopy with a mucosal flap (SEMF): a short tunnel is created by additional submucosal dissection to approach the lesion. $\mathbf{d}$ Endoscopic image of the whitish tumor identified through the tunnel. e Core biopsy: a core specimen measuring $5 \times 5 \times 2 \mathrm{~mm}$ is obtained using a needle knife. $\mathbf{f}$ Tissue collection into a transparent cap: the specimen is removed into a long attachment using grasping forceps. $\mathbf{g}$ Clip closure of the flap: The opening flap is completely closed with hemoclips. h Endoscopic image showing the clip closure.

tal Medical Information Network Clinical Trials Registry Number UMIN 000006754 following the CONSORT check list. All patients provided written informed consent to undergo the procedures and participate in the study.

\section{Submucosal tunneling biopsy}

All patients were placed under deep sedation wth intravenous midazolam $(0.05 \mathrm{mg} / \mathrm{kg})$. STB consisted of 5 major procedures $(\triangleright$ Fig. 2$)[17,18]$. All procedures of STB are presented in $\downarrow$ Video 1 . In the first step, creation of the entry, the endoscopic submucosal dissection (ESD) technique was introduced after marking 2 dots around the lesion at a margin of approximately $5 \mathrm{~mm}$, with 1 dot at the top of the lesion. A 10-mm entry was created by a mucosal incision using a submucosal injection of $0.4 \%$ hyaluronate sodium solution (MucoUp; Johnson \& Johnson K.K., Tokyo, Japan) with a needle knife (KD-441Q; Olympus, Tokyo, Japan) ( $\mathbf{F i g . 2 a}$ and $\triangleright$ Fig. 2 b). In the second step, SEMF, a short tunnel with an opening flap was created by submucosal dissections toward the lesion ( $\mathbf{F i g . 2 c}$ ). In the third step, the core biopsy, after the lesion was visualized through the tunnel ( $\triangleright$ Fig. $\mathbf{2 d}$ ), a core specimen with a maximum diameter of approximately $5 \mathrm{~mm}$ was acquired using the needle knife in cutting mode on the electrosurgical unit (VIO300 D, EndoCut mode effect 2, duration 3; ERBE Elektromedizin, Tübingen, Germany) while minimizing tissue crushing ( $\triangleright$ Fig. 2 e). If this step appeared to be technically difficult, biopsy forceps (Radial $J^{\text {JM }} 4$ Standard Capacity; Boston Scientific, Tokyo, Japan) were introduced, adding 1 break for the lesion (approximately $2 \mathrm{~mm}$ in diameter) with the needle knife, which aimed at avoiding slippage due to tumor rigidity when grasping the biopsy forceps. In the fourth step, tissue collection, the specimen was separated from the lesion with grasping forceps (FG-6U-1; Olympus) or biopsy forceps and collected into a transparent cap with longer characteristics at the tip (Elastic Touch F-01, Top Corporation, Tokyo, Japan) ( $\triangleright$ Fig. 2f). Special care was taken to prevent contact of the tissue with the inner wall of the tunnel. In the final step, clip closure, the entry was sutured with hemoclips ( $\mathbf{F i g .} \mathbf{2 g}$ and $\nabla$ Fig. $\mathbf{2 h}$ ). Bleeding was mana-

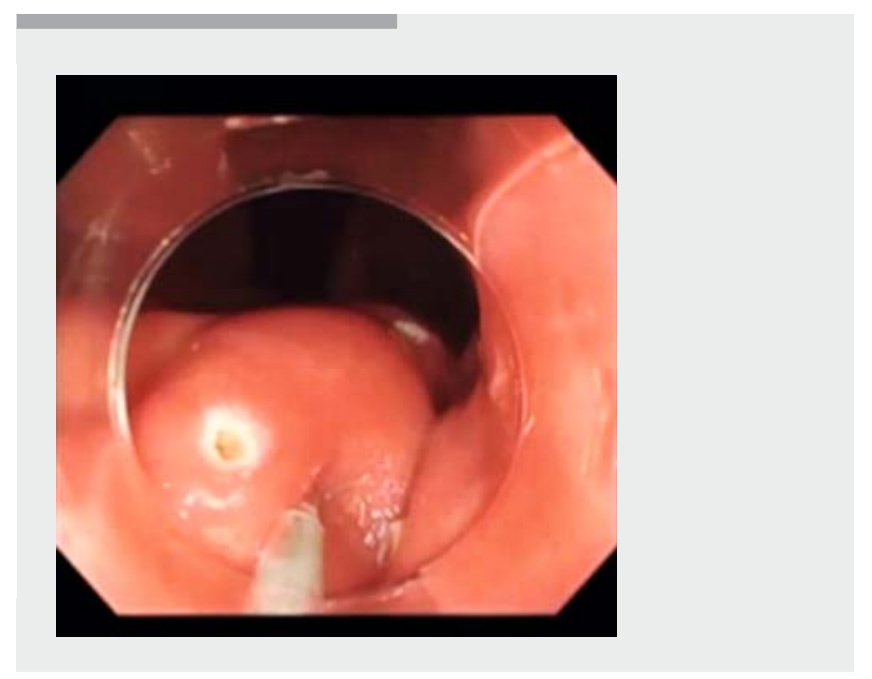

Video 1 Submucosal tunneling biopsy (STB) for a gastric subepithelial lesion. While creating a short submucosal tunnel via a 10 -mm entry, a whitish tumor is exposed and visualized. After tissue sampling, the entry is completely closed with hemoclips. Histological examination confirmed a leiomyoma. 
ged in all procedures using hemostatic forceps (FD-410 LR; Olympus).

In the case presented in this video, the IH analysis revealed cKIT negative, $\alpha$-smooth muscle actin and desmin-positive tissue, confirming a diagnosis of gastric leiomyoma. All procedures were performed by a single endoscopist (H.K.) who has successful experience with more than 200 gastric ESD cases.

\section{Endoscopic ultrasound-guided fine needle aspiration}

With patients in the left lateral position under deep sedation, FNA was performed using a conventional convex scanner echoendoscope (UCT-240-AL5; Olympus) connected to an ultrasound scanner (ProSound SSD- $\alpha 10 ;$ ALOKA, Tokyo, Japan). The puncture was performed with disposable 22 - to 25-gauge aspiration needles, followed by 19-gauge needle (Expect $^{\mathrm{TM}}$ standard type; Boston-Scientific, Tokyo, Japan). Color flow mapping was applied to avoid puncturing vessels.

FNA was performed as described [19]. Briefly, after advancing the needle into the lesion under EUS visualization, the stylet was removed. Next, suction was applied with a $10-\mathrm{mL}$ syringe as the needle was moved backward and forward within the lesion. The needle was moved in various directions more than 10 times within the lesion during each puncture session. After the entire catheter was removed, the aspirated specimens were transferred to a Petri dish containing saline solution. The aspiration procedure was repeated until whitish tissue appeared macroscopically, with a maximum of 5 passes. On-site pathologists were present to determine adequacy of specimens. All FNA procedures were performed by an experienced endosonographer (H.K.) who has successfully performed more than 200 FNA procedures. That endosonographer received FNA training at a high-volume center in Japan and gained experience with 30 cases of gastric SEL before starting our study.

\section{Treatment protocol}

Patients were hospitalized for 6 days. Informed consent was reconfirmed on Day 1 , the first procedure (STB or FNA) was performed on Day 2, and the second procedure (FNA or STB) was performed on Day 4. Patients were discharged on Day 6 after undergoing these procedures. We assessed the laboratory data on postoperative Days 3 and 5 . Upon discharge, patients were surveyed for 30 days to record late AEs.

\section{Pathological assessment of the sample}

Pathology diagnosis was made based on hematoxylin and eosin (H\&E) staining and immunohistochemical stains. GIST risk stratification of malignancy (very low, low, moderate, high) was classified based on Fletcher classification [20]. Final diagnosis was categorized as diagnostic or non-diagnostic (defined to include suspicious and atypical readings) with sampling tissues and/or surgically resected specimens and was standardized among 3 experienced pathologist (R. H. and 2 experienced pathologists).

\section{Outcome measures}

The primary outcome measure was comparison of total diagnostic yield (DY) from STB and FNA in Group A and B. The diagnostic yield was defined as the percentage of lesions confirmed by a pathologic diagnosis involving immunohistological analysis. Secondary outcome measures were technical success rate, procedure time, biopsy frequency of technically successful cases, rate of complications, and sample quality of obtained specimen. Technical success was defined as accessing the target tissue and obtaining visible tissue specimens or fragments; technical failure included no tissues despite successful needle puncture of the lesion, the inability to maneuver the endoscope, and absence of an access route, including inability of the needle to exit the channel at the scope tip because of the angle. Procedure time for FNA was defined as the time from inserting the needle into the scope channel until its removal, whereas procedure time for STB was defined as the time from the start of marking until clip closure. Biopsy frequency was defined as the number of times the endoscopist biopsied the target lesion. Complication was defined as the occurrence of perforation and bleeding requiring a blood transfusion proved by endoscopic, CT, and blood examinations during the procedure, hospitalization, and a 30-day survey. Regarding the quality of acquired samples, the length (major $\times$ minor, $\mathrm{mm}$ ) and the overlay area $\left(\mathrm{mm}^{2}\right)$ of each piece of specimen from each sampling method were measured using digital imaging software (cellSens Standard; Olympus, Tokyo, Japan).

\section{Sample size calculation}

Based on previous studies [13-15], the diagnostic accuracy rate of FNA for SEL was assumed to be $50 \%$. We further assumed that STB afforded a diagnostic accuracy of $90 \%$ according to our previous study [18]. A 2-tailed sample size calculation was performed assuming a type I error rate of 0.05 , and a power of $80 \%$ for detecting a difference in the diagnostic yield between STB and FNA. Assuming a $10 \%$ drop-out rate, we calculated a final sample size of 42 patients ( 21 per group).

\section{Statistical analysis}

Continuous data are presented as medians and ranges. The McNemar test was used to compare the DY and technical success rate of STB and FNA. We calculated the $95 \%$ confidence interval $(\mathrm{Cl})$ of the DY difference between STB and FNA using Newcombe's procedure because each method was observed in a paired case [21]. Procedure time and biopsy frequency were compared between both methods using 2-sided Wilcoxon signed-rank tests. Major length and overlay area of each piece of specimen were compared between both methods using a paired $t$-test. The $95 \% \mathrm{Cls}$ were calculated for the DY of STB and FNA based on the Agresti-Coull procedure. The DYs of the 2 methods in relation to each parameter were compared using 2 -sided Fisher's exact tests. $P<0.05$ was considered statistically significant. All statistical analyses were conducted using JMP 11.2.0 (SAS Institute Inc., Cary, NC, USA). 
- Table 1 Patient demographics and gastric subepithelial lesion characteristics.

\begin{tabular}{|c|c|}
\hline \multicolumn{2}{|l|}{ Characteristics } \\
\hline Total number of patients & 43 \\
\hline Sex, Male/Female, n. & $20 / 23$ \\
\hline Median age (range), y & $66(40-88)$ \\
\hline \multicolumn{2}{|l|}{ Location (stomach), n. } \\
\hline - Upper & 23 \\
\hline - Middle & 13 \\
\hline - Lower & 7 \\
\hline Lesion maximum size on EUS, median (range), mm & $15(7-45)$ \\
\hline . $<2 \mathrm{~cm}, \mathrm{n}$ & 29 \\
\hline . $\geq 2 \mathrm{~cm}, \mathrm{n}$ & 14 \\
\hline \multicolumn{2}{|l|}{ EUS finding, $\mathrm{n}$} \\
\hline \multicolumn{2}{|l|}{ Layer in origin } \\
\hline - Submucosa & 6 \\
\hline - Muscularis propria & 37 \\
\hline \multicolumn{2}{|l|}{ Echoic pattern } \\
\hline - Hyро & 36 \\
\hline - Hyper & 1 \\
\hline - Mixed & 6 \\
\hline
\end{tabular}

\section{Results}

\section{Patient characteristics}

Of 57 patients identified as potentially eligible for participation during the study period, 14 were excluded for the following reasons: obvious cases of 1 lipoma and 2 simple cysts on EUS $(n=3)$, SELs with extraluminal growth $(n=6)$, lesion size $>5 \mathrm{~cm}$ $(n=3)$, and patients who declined to participate $(n=2)$. Accordingly, 43 consecutive patients (20 men, 23 women, median age: 66 years, range: $40-88$ years) were enrolled and analyzed.

Their detailed clinical data are summarized in $>$ Table 1 . The characteristics of all regions, layer of origin, lesion size, and echo density were evaluated by EUS. Of the 43 SELs, 23 were located in the upper stomach, 13 were located in the middle stomach and 7 were located in the lower stomach. Median lesion size of the lesions was $15 \mathrm{~mm}$ (range 7-45), consisting of 29 cases with $<2 \mathrm{~cm}$ and 14 cases with $\geq 2 \mathrm{~cm}$. EUS demonstrated that 37 SELs originated in the muscularis propria (MP) layer and 6 SELs originated in the submucosal layer.

\section{Primary and secondary outcomes}

A flow diagram of the study results is shown in $>$ Fig. 3. Primary and secondary outcomes are summarized in $>$ Table 2. Twenty subjects of FNA were missing in Group B (STB $\rightarrow$ FNA) due to carry-over effects by STB. Thus, data from group A were the focus of the analyses.

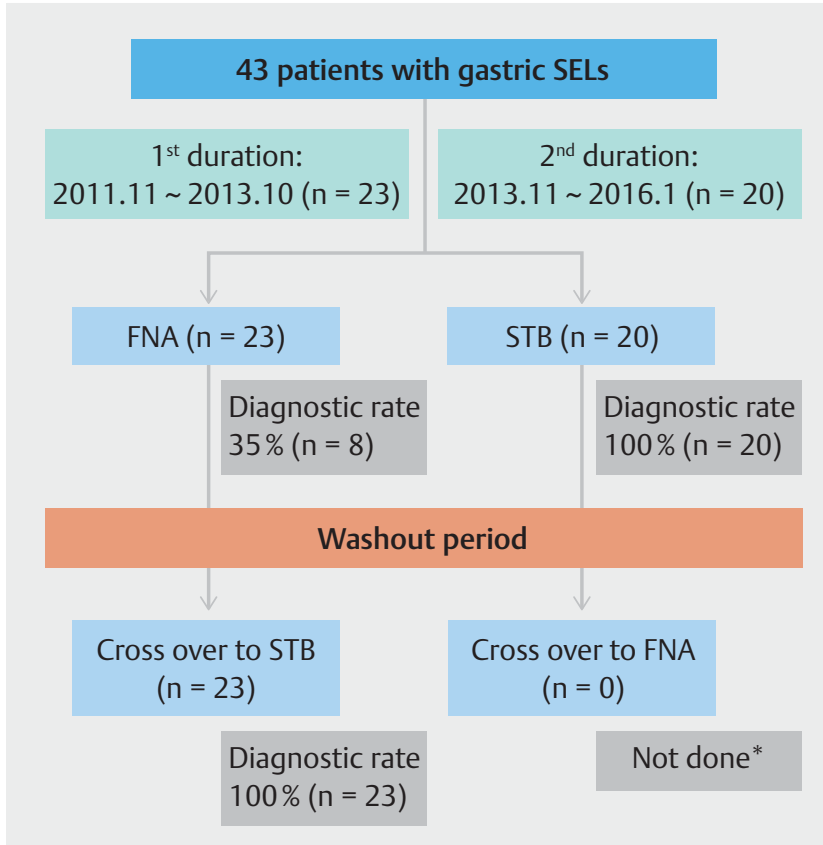

- Fig. 3 Flow diagram of study results. *FNA could not proceed because several hemoclips used by STB induced poor EUS images due to acoustic artifact.

The DY of STB was significantly higher than that of FNA (23/ $23,100 \%$ vs. $8 / 23,34.8 \% ; P<0.0001)$ in Group A. The difference in DY was $65.2 \%(95 \% \mathrm{Cl}=38.7$ to $81.3 \%)$. The $95 \% \mathrm{Cls}$ with the DY were $18.7-55.2 \%$ for FNA, $83.1-100 \%$ for STB, and $90.2-100 \%$ for overall STB $(n=43)(\triangleright$ Table 3$)$. Technical success rates were $56.5 \%$ (13/23) for FNA and $100 \%(23 / 23)$ for STB $(P<0.05)$. DY and failure factors of FNA are summarized in Table 4. When technically successful, median procedure times were 18 minutes ( $n=13$; range, $13-34$ ) for FNA and 37 minutes $(n=23$; range $19-90)$ for STB $(P<0.05)$, and median biopsy frequencies were 3 times for FNA $(n=13$; range $2-5)$ and 1 time for STB $(n=23$; range $1-1)(P<0.05)$. No complications occurred during or after either procedure.

\section{Quality of tissue samples}

Thirty-one samples obtained by FNA and STB (FNA, 8 samples; STB, 23 samples) in Group A were histologically evaluated. The medians of the length (major $\times$ minor) and overlay area of the obtained specimens were $1.8 \mathrm{~mm}$ (range $0.71-2.8$ ) $\times 0.47 \mathrm{~mm}$ (range $0.4-0.8$ ) and $0.69 \mathrm{~mm}^{2}$ (range $0.16-1.81$ ) for FNA in 8 immunohistologically successful cases and $3.9 \mathrm{~mm}$ (range $2.0-6.4) \times 2.5 \mathrm{~mm}$ (range $1-4.2$ ) and $5.54 \mathrm{~mm}^{2}$ (range $1.86-$ 12.1 ) for STB in 23 cases $(P<0.05)$, respectively ( $>$ Fig. $4 a$ and - Fig. 4b). A representative tissue fragment with H\&E staining at the same magnification $(\times 12.5)$ in one GIST low-risk case sampled by FNA and STB is displayed in > Fig. 5 a and $>$ Fig. $\mathbf{5 b}$, respectively. The STB specimen was sufficiently large for $\mathrm{IH}$ analysis, whereas the FNA biopsy specimen was small and contained in a blood clot. 
- Table 2 Comparison of outcomes of EUS-FNA (FNA) and STB for gastric SELs.

\begin{tabular}{|c|c|c|c|c|c|}
\hline & & $\begin{array}{l}\text { FNA } \\
(n=23)\end{array}$ & $\begin{array}{l}\text { STB } \\
(n=23)\end{array}$ & $P$ value & $\begin{array}{l}\text { Treatment } \\
\text { Difference } \\
\left(95 \% \mathrm{Cl}^{1}\right)\end{array}$ \\
\hline \multirow[t]{2}{*}{$\begin{array}{l}\text { Primary } \\
\text { outcomes }\end{array}$} & $\begin{array}{l}\text { Diagnostic yield } \\
\text { (Final definitive diagnosis involving } \\
\text { immunohistological analysis), \%, (n) }\end{array}$ & $34.8(8 / 23)$ & $100(23 / 23)$ & $<0.0001^{2}$ & $65.2 \%$ (38.7 to 81.3 ) \\
\hline & Complication rate, $\%$ & None & None & & \\
\hline \multirow{3}{*}{$\begin{array}{l}\text { Secondary } \\
\text { outcomes }\end{array}$} & Technical success rate, $\%,(n)$ & $56.5(13 / 23)$ & $100(23 / 23)$ & $0.0006^{2}$ & \\
\hline & Procedure time, median (range), min & $\begin{array}{l}18(13-34) \\
(n=13 \text { technically successful) }\end{array}$ & $37(19-90)$ & $<0.0001^{3}$ & \\
\hline & $\begin{array}{l}\text { Biopsy frequency, median (range), } \\
\text { times } \\
\text { Overlay area of acquired specimen, } \\
\text { median (range), } \mathrm{mm}^{2}\end{array}$ & $\begin{array}{l}3(2-5) \\
(n=13 \text { technically successful) } \\
0.69(0.16-1.81) \\
\text { ( } n=8 \text { Immunohistologically } \\
\text { successful) }\end{array}$ & $\begin{array}{l}1(1-1) \\
5.54(1.86-12.1)\end{array}$ & $\begin{array}{l}<0.0001^{3} \\
<0.001^{4}\end{array}$ & \\
\hline \multicolumn{6}{|c|}{$\begin{array}{l}\text { EUS-FNA, endoscopic ultrasound-guided fine needle aspiration; SEL, subepithelial lesions; STB, submucosal tunneling biopsy. } \\
195 \% \text { confidence interval (CI) for the difference between binomial proportions based on paired data } \\
{ }^{2} \text { McNemar test } \\
{ }^{3} \text { Wilcoxon signed-rank test, two-sided } \\
{ }^{4} \text { paired t-test }\end{array}$} \\
\hline
\end{tabular}

- Table 3 Diagnostic yield and 95\% confidence interval of FNA and STB for gastric SELs.

\begin{tabular}{|l|c|c|}
\hline & Diagnostic yield (\%) & $\mathbf{9 5 \% ~ C l ~ ( \% )}$ \\
\hline FNA $(n=23)$ & 34.8 & $18.7-55.2$ \\
\hline STB $(n=23)$ & 100 & $83.1-100$ \\
\hline overall STB $(n=43)$ & 100 & $90.2-100$ \\
\hline
\end{tabular}

FNA, fine-needle aspiration; SEL, subepithelial lesion; STB, submucosal tunneliing biopsy

${ }^{1} 95 \% \mathrm{Cl}$ was based on the Agresti-Coull procedure.

\section{Failure factor of FNA}

Technical failure of FNA occurred because no tissue was collected from 6 patients, no platform was available in 2 patients, and there was no access route in 2 patients. In the 5 patients from whom tissue samples were acquired successfully using FNA, samples were not suitable for IH analysis (insufficient material); of these patients, 1 was suspected of having spindle cells, whereas the other 4 were not evaluated.

The DYs of FNA and STB were compared with regard to location, lesion size, and diagnosis ( $\vee$ Table 5 ). Lesions in the upper and lower stomach were associated with inadequate tissue yield by FNA. The DYs for FNA and STB of lesions $<2 \mathrm{~cm}$ in size, of GISTs, and of leiomyomas differed significantly $(P<0.05)$. Other factors could not be assessed statistically because of the small sample sizes.

\section{Histopathologic examination}

IH analysis of specimens obtained by STB confirmed 20 GISTs (8 very-low-risk, 11 low-risk, and 1 moderate according to Fletcher classification), 12 leiomyomas, 3 heterotopic pancreases, 2 schwannomas, 2 lipomas, 2 duplication cysts, 1 granular cell tumor, and 1 amyloidosis. Seventeen of 20 patients with gastric GIST underwent surgery, although 2 patients with very-low-risk GISTs rejected additional surgery because of advanced age (both aged 82 years) and 1 patient with low-risk GIST is scheduled to undergo surgery.

The IH correlation rate between STB specimens and resected surgical specimens in all 17 resected patients was $100 \%$. All patients with GIST underwent surgical local resection, including submucosal tunnel and the entry created by STB, to accurately identify the horizontal margin of the tumor. Pathologic examination revealed that no tumor cells in any of these patients were disseminated in the submucosal tunnels and superficial epithelium of resected specimens ( Fig.6a and $>$ Fig.6b). None of these 17 patients experienced tumor recurrence during a mean follow up of 29.5 months (range, 3-50 months).

\section{Discussion}

This study is the first prospective, comparative study with crossover design that compared diagnostic yield of novel STB and EUS-FNA in patients with endoluminal gastric SELs. In the current study, although lesions $<2 \mathrm{~cm}$ comprise more than half of all cases, we identified 2 important clinical findings. First, the accuracy rate of IH analysis and technical success in STB were significantly higher than those in FNA without procedure-related complications, even for small SELs. Second, STB was superior to FNA in terms of obtaining samples sufficient for histological interpretation.

EUS-FNA is the current standard method for sampling gastrointestinal SELs [4-8]. Nevertheless, a systematic review with a meta-analysis of 17 studies indicated a moderate diagnostic accuracy of $59.9 \%(95 \% \mathrm{Cl}, 54.8-64.7)$ in 978 attempts 
- Table 4 Diagnostic yield and failure factors of FNA in patients with gastric SELs $(n=23)$.

\begin{tabular}{|c|c|c|}
\hline & Technical success with an acquired specimen & Technical failure without an acquired specimen \\
\hline Number of patients, $\mathrm{n}(\%)$ & $13(56.5)$ & $10(43.5)$ \\
\hline \multicolumn{3}{|l|}{ Diagnosis, n (\%) } \\
\hline - Diagnostic & $8(34.8)$ & $0(0)$ \\
\hline - Non-diagnostic & $5(21.7)$ & $10(43.5)$ \\
\hline \multicolumn{3}{|l|}{ Failure factor, $\mathrm{n}$} \\
\hline - Insufficient material & $5^{1}$ & \\
\hline - No tissue & & 6 \\
\hline - No route & & $2^{2}$ \\
\hline - No platform & & 2 \\
\hline \multicolumn{3}{|c|}{$\begin{array}{l}\text { FNA, fine-needle aspiration; GIST, gastrointestinal stromal tumor; SEL, subepithelial lesion } \\
\text { No tissue: no tissues despite successful needle puncture of the tumor, No route: lack of access route, including the inability of the needle to exit the channel at the } \\
\text { scope tip because of the angle, No platform: inability to maneuver the endoscope. } \\
{ }^{1} \text { suspicious; spindle cells in one case } \\
2 \text { Location; cardia } 2 \text { cases }\end{array}$} \\
\hline
\end{tabular}
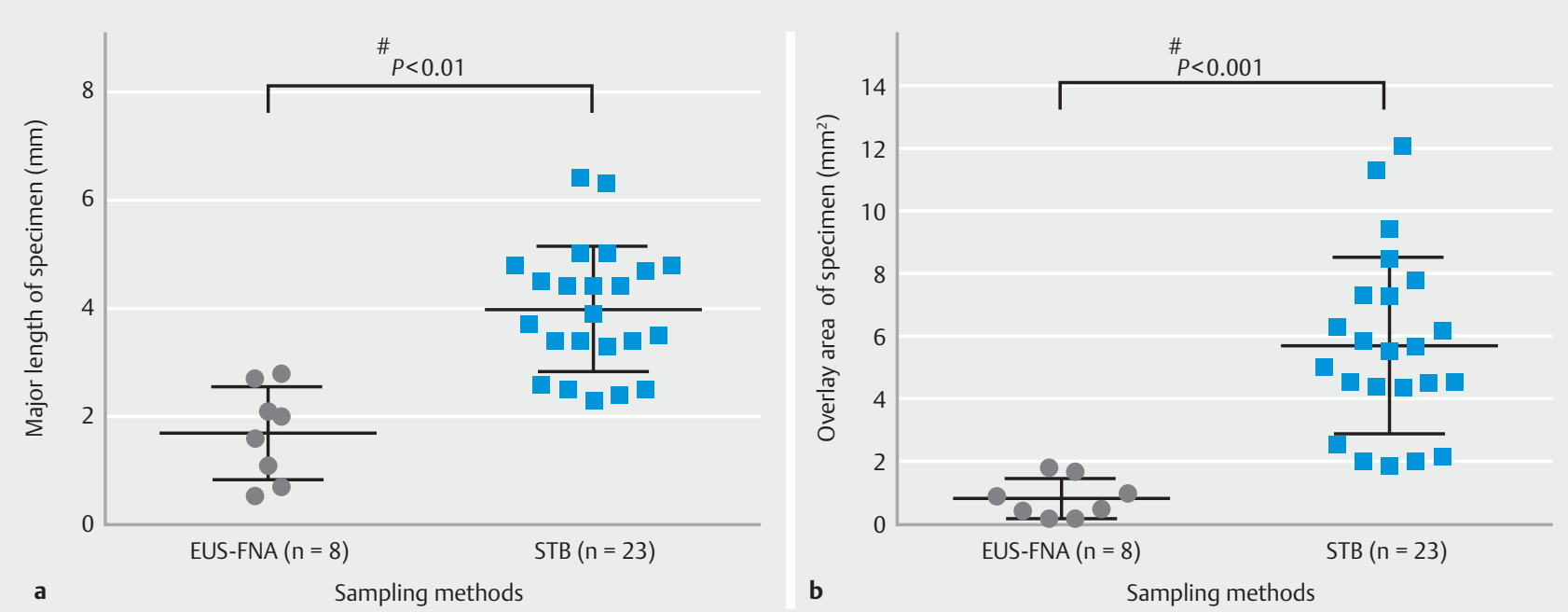

- Fig. 4 Plot showing individual measurements of the major size and overlay area of the specimen: comparison of FNA and STB samples. a Median major length of the acquired specimens was $1.8 \mathrm{~mm}$ (range, $0.71-2.8$ ) for FNA in 8 immunohistologically successful cases and $3.9 \mathrm{~mm}$ (range, $0.71-2.8$ ) for STB in 23 cases $(P<0.05)$. b Median overlay area of the acquired specimens was $0.69 \mathrm{~mm}^{2}($ range, $0.16-1.81)$ for FNA in 8 immunohistologically successful cases and $5.54 \mathrm{~mm}^{2}$ (range, $\left.1.86-12.1\right)$ for STB in 23 cases $(P<0.05)$.

" paired $t$-test

of FNA sampling for upper GI SELs [22]. Failures of FNA occur due to the insufficient materials for IH analysis, technical problems, location, and lesions $<2 \mathrm{~cm}$. A recent study concluded that a lesion size $<2 \mathrm{~cm}$ was an independent failure factor for FNA based on multivariate analysis [23].

In the current study, the diagnostic yield of FNA sampling $(34.8 \%)$, which is defined as a definitive final diagnosis involving immunohistological analysis, may sound too poor.

Although other studies reported that overall diagnostic rates including cytological examination were satisfactory with $83.9 \%$ $(n=112)$ [13], and $82.3 \%(n=141)$ [14], definitive final diagnostic yields were still unsatisfactory with $61.6 \%$, and $43.3 \%$, respectively. Moreover, Fernández-Esparrach $G$ reported that the overall diagnostic accuracy of FNA was $52 \%$ and that of trucut biopsy (TCB) was 55\% [15]. Accordingly, our results with FNA might be compatible with results from other studies. In addition, our endosonographer, who previously demonstrated similar diagnostic ability withr 30 cases of gastric SEL before the study, estimated his skill as almost standard level.

Technical issues were caused by difficulty in maneuvering the endoscope in 10 of 23 patients (technical error rate, $43.5 \%$ ). In the 5 patients from whom no tissue was obtained, the procedure failed due to small and mobile lesions despite successful needle puncture. Another factor was the location of the lesion in the 

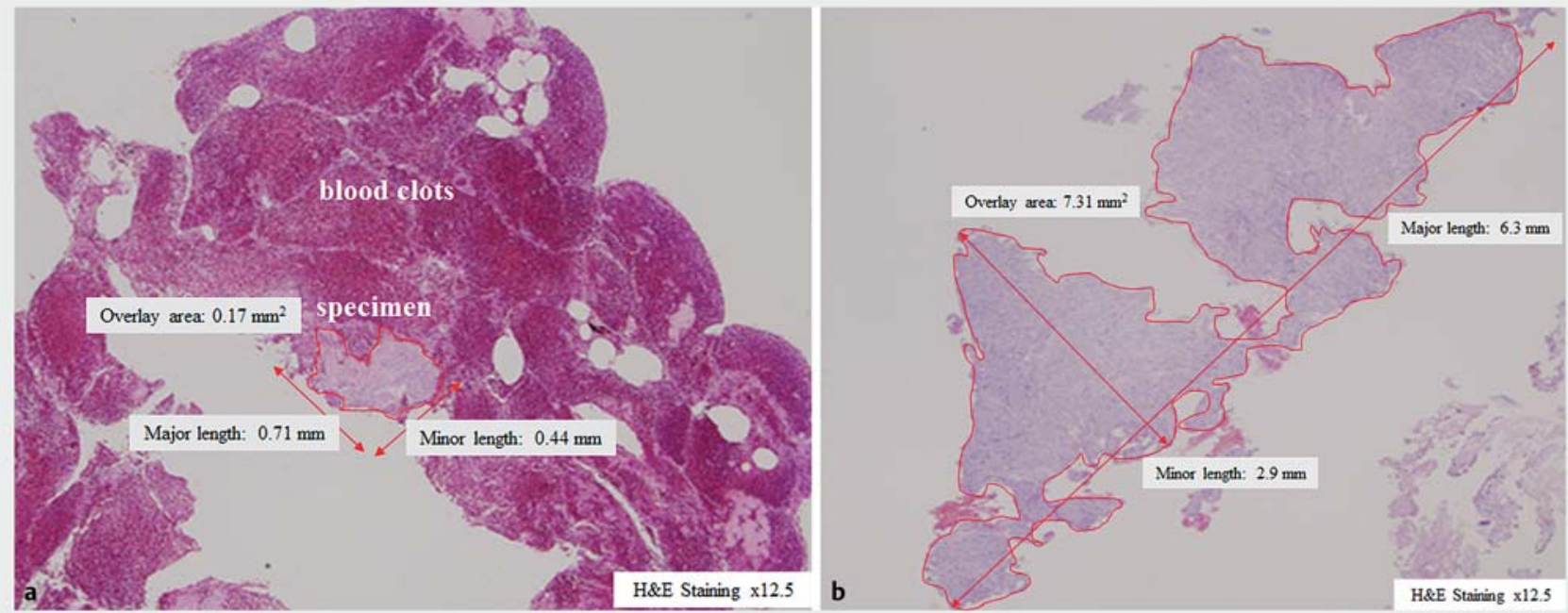

- Fig. 5 Comparison of tissue quantities obtained by FNA and STB from a low-risk GIST at the same magnification $(\times 12.5)$ following H\&E staining. a The FNA biopsy specimen obtained with the 22-gauge FNA needle was small in terms of length (major $\times$ minor; $0.71 \times 0.44 \mathrm{~mm}$ ) and overlay area $\left(0.17 \mathrm{~mm}^{2}\right)$ and occupied in blood clots. $\mathbf{b}$ The specimen acquired by STB was of sufficient size in terms of length (major $\times$ minor; $6.3 \times 2.9 \mathrm{~mm})$ and overlay area $\left(7.31 \mathrm{~mm}^{2}\right)$.

- Table 5 Comparison of definitive diagnostic yields on FNA and STB in relation to each measured parameter

\begin{tabular}{|c|c|c|c|c|}
\hline \multirow[b]{2}{*}{ Parameter } & \multirow[t]{2}{*}{ Methods } & FNA $(n=23)$ & STB $(n=43)$ & \multirow[b]{2}{*}{ Pvalue $^{1}$} \\
\hline & & \multicolumn{2}{|c|}{$\%$ (n, success/intervention) } & \\
\hline \multirow[t]{3}{*}{ Location (stomach) } & $U$ & $40 \%(6 / 15)$ & $100 \%(23 / 23)$ & 0.0007 \\
\hline & M & $50 \%(2 / 4)$ & $100 \%(13 / 13)$ & 0.4286 \\
\hline & $\mathrm{L}$ & $0 \%(0 / 4)$ & $100 \%(7 / 7)$ & 0.0286 \\
\hline \multirow[t]{2}{*}{ Lesion maximum size } & $<2 \mathrm{~cm}$ & $35.3 \%(6 / 17)$ & $100 \%(29 / 29)$ & $<0.0001$ \\
\hline & $\geq 2 \mathrm{~cm}$ & $33.3 \%(2 / 6)$ & $100 \%(14 / 14)$ & 0.0606 \\
\hline \multirow[t]{8}{*}{ Diagnosis (n) } & GIST (20) & $50 \%(6 / 12)$ & $100 \%(20 / 20)$ & 0.0137 \\
\hline & Leiomyoma (12) & $20 \%(1 / 5)$ & $100 \%(12 / 12)$ & 0.0476 \\
\hline & Heterotopic pancreas (3) & $0 \%(0 / 2)$ & $100 \%(3 / 3)$ & 0.3333 \\
\hline & Lipoma (2) & $0 \%(0 / 1)$ & $100 \%(2 / 2)$ & - \\
\hline & Schwannoma (2) & $0 \%(0 / 1)$ & $100 \%(2 / 2)$ & - \\
\hline & Duplication cyst ( 2 ) & $0 \%(0 / 1)$ & $100 \%(2 / 2)$ & - \\
\hline & Granular cell tumor (1) & $100 \%(1 / 1)$ & $100 \%(1 / 1)$ & - \\
\hline & Amyloidosis (1) & - & $100 \%(1 / 1)$ & - \\
\hline
\end{tabular}

upper and lower stomach, likely because it is difficult to handle the scope and needle device smoothly in this area due to strong flexion of the scope.

Further attempts are needed to improve the diagnostic yield of FNA for SELs. Use of softer needles such as Echotip Ultra (Cook Medical, Tokyo, Japan) may be effective for a difficult situation due to strong flexion of the scope. Individualized choice of needle devices according to the situation and lesion size may resolve technical problems. Moreover, introduction of rapid onsite evaluation (ROSE) can lead to the decision whether an FNA procedure should be finished or not after confirmation of the adequacy of acquired specimens [24].

Hence, we developed a novel STB to overcome these issues and increase the DY. Considering the limitation of FNA for small SELs, the frequently encountered type in clinical practice, SELs $<2 \mathrm{~cm}$ were included at a high ratio $(67.4 \%, 29 / 43)$ in our study. 

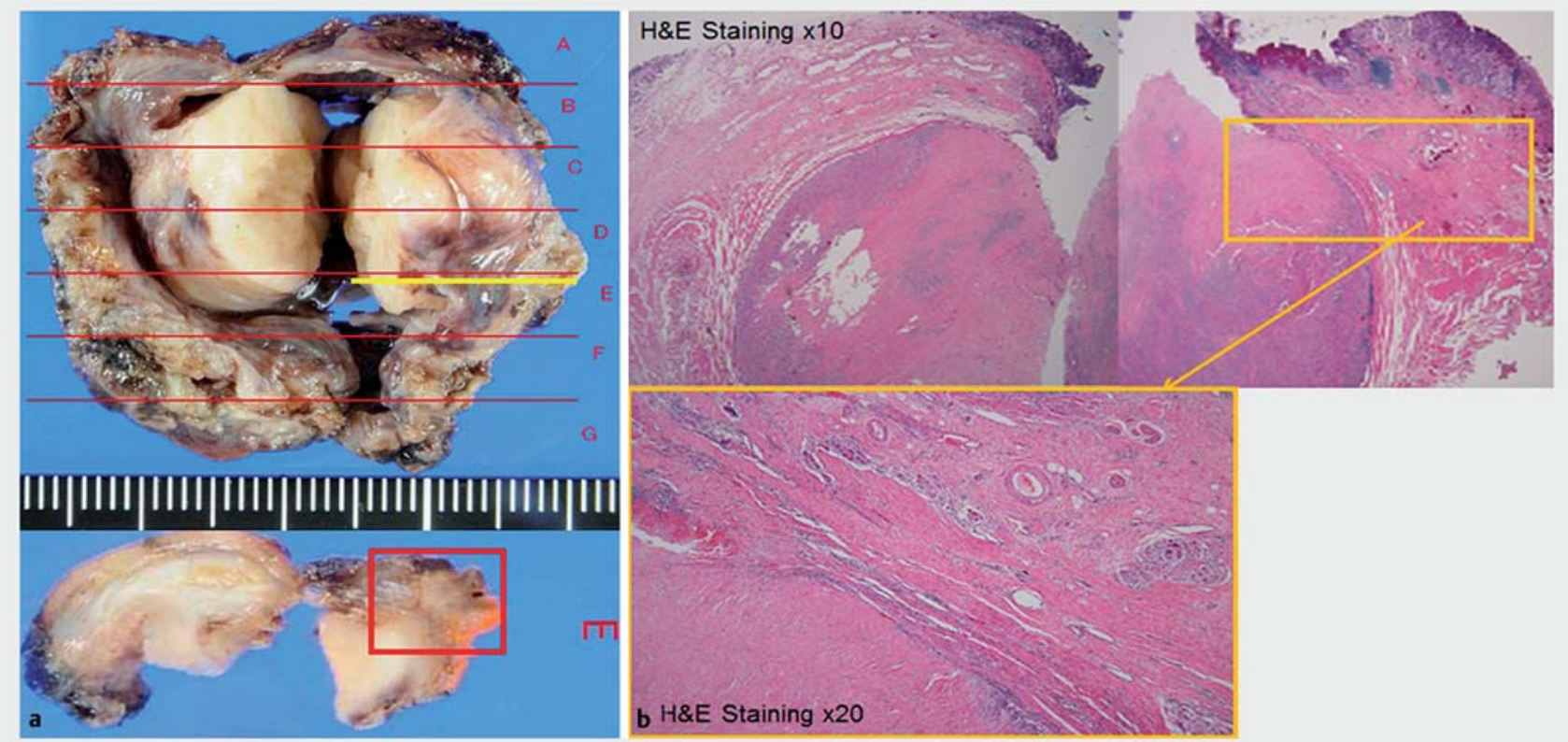

- Fig. 6 Histological findings of submucosal tunnels dissected by STB for gastrointestinal stromal tumors (GISTs) ( $n=17)$. Histologically, none of the 17 GISTs that underwent surgical local resection, including simultaneous resection of the tunnel, showed tumor seeding within the short tunnel. Only fibrotic tissue was visible in the tunnel. a Macroscopic findings of a low-risk GIST, showing fibrosis (yellow line) and the cut surface of the short tunnel (red box). b Histologically, there was no evidence of dissemination of tumor cells into the submucosal tunnel dissected by STB (H\&E staining; magnification, $\times 10$ and $\times 20$ ).

According to our result for SELs $<2 \mathrm{~cm}$, STB had a significantly different DY from FNA (100\% for STB vs. 35.3\% for FNA). These data suggested that STB can offer important diagnostic advantages over FNA, which has limited accuracy for small SELs. In addition, FNA required multiple punctures (mean biopsy frequency, 2.9 times) and exchange of puncture needles because of inadequate tissue sampling, whereas a single tissue block obtained by STB was adequate for IH analysis. According to a major guideline, a tissue sample area of approximately $4.8 \mathrm{~mm}^{2}$ is necessary for evaluating the risk classification of GIST involving mitotic count/50 HPF (high-power field) [6]. However, conventional sampling methods often fail in preoperative diagnosis of GIST risk classification due to small amounts of tissue sample. In contrast, here, STB provided larger amounts of pure specimens without contamination than FNA containing blood clots (median sample area, $5.54 \mathrm{~mm}^{2}$ for STB vs. $0.69 \mathrm{~mm}^{2}$ for FNA). When a tissue amount does not meet the assessment of mitotic count/50HPF, substitution of Ki-67 (M1B labeling) index would be suitable for preoperative diagnosis of GIST risk classification [25]. These pure and sufficient STB specimens are based on the STB advantages of identifying the lesion itself [26] and obtaining core specimens through the submucosal tunnel. Moreover, this strength of STB can lead to novel translational research [27].

In contrast, STB has several disadvantages. First, procedure time was more than twice as long as that for FNA (median, $37 \mathrm{~min}$ vs. $18 \mathrm{~min}$ ). Of all the STB steps, obtaining a fusiform tissue block $(5 \mathrm{~mm})$ with a knife at the tip in the narrow submucosal tunnel required not just skill but also time. STB based on ESD technique is more invasive than FNA using a thin needle. Moreover, FNA done conveniently even at an outpatient clinic is superior to STB in terms of cost benefits including used devices (FNA vs. STB: 245 vs 551 USD) and hospitalization. Thus, convenient FNA may be a reasonable first approach for SELs sampling, followed by STB if necessary.

In addition, STB will likely result in significant submucosal fibrosis, which may make future endoscopic tissue sampling and resection attempts difficult [28]. Combining STB with another tool may be optimal. For example, endoscopically visualized features of the lesion itself [26] may determine whether the lesion is resectable or should be conserved in a single approach.

EUS-FNA is considered a relatively safe method, with complications, including bleeding and infection, being rare [15]. In this study, none of our patients experienced any complications during or after either procedure. The result of STB prove that clip closure based on SEMF can be helpful for preventing delayed complications.

STB uses tissue collection into a transparent cap, clip closure of the flap, and simultaneous resection of the tunnel during local surgical resection to prevent tumor seeding. Histologically, none of the 17 GISTs for which surgery was performed exhibited tumor seeding within the short tunnel, suggesting that tumor cells would not implant into the gastric lumen. Measures are also taken to prevent an accidental perforation when obtaining tissue samples.

This study had several limitations. One limitation of STB use is the requirement for an experienced endoscopist who is familiar with ESD techniques. The inclusion criteria involve only en- 
doluminal gastric SELs. Patient enrollment was not assigned as a randomized trial. After we verified the invasiveness and safety of STB at the Group A due to lack of safety data on a novel STB, we planned to proceed to Group B.

Data on FNA are insufficient because of carry-over effects by STB in Group B. After the first patient in Group B underwent successful STB procedures, FNA resulted in failure because several hemoclips used during STB remained around the lesion, resulting in poor EUS images due to acoustic artifact. Accordingly, we were forced to modify the protocol for Group B as follows: a crossover to FNA was applied only if STB failed in the pathological diagnosis, providing a washout period longer than 2 weeks. As a result, because STB in Group B provided perfect outcomes with a diagnostic yield of $100 \%$, FNA was not performed in that group. This resulted in lack of data about FNA in Group B. So, when the results of both stages were analyzed using permutated distribution to confirm the internal validity of obtained results, it was considered that there was little influence in regard to the bias due to background factor.

\section{Conclusion}

In conclusion, this study demonstrated that the STB technique had a significantly higher diagnostic ability than FNA for gastric subepithelial lesions, even those $<2 \mathrm{~cm}$ in diameter, which could guide therapeutic planning.

\section{Acknowledgements}

This work was supported in part by UEG Top 5 Abstract Prize2013 to Hideki Kobara. This work was supported by Japan Society for the Promotion of Science (JSPS) KAKENHI Grant Number 15K06855. Additionally, this work was supported by Alumni Association of Faculty of Medicine, Kagawa University, Grant Number 27-1. These funders had no role in study design, data collection or analysis, decision to publish, or preparation of the manuscript.

\section{Competing interests}

None

References

[1] Hwang JH, Kimmey MB. The incidental upper gastrointestinal subepithelial mass. Gastroenterology 2004; 126: 301-307

[2] Blay J-Y, Bonvalot S, Casali P et al. Consensus meeting for the management of gastrointestinal stromal tumors Report of the GIST Consensus Conference of 20-21 March 2004, under the auspices of ESMO. Ann Oncol 2005; 16: 566 - 578

[3] Mori H, Kobara H, Kobayashi M et al. Establishment of pure NOTES procedure using a conventional flexible endoscope: review of six cases of gastric gastrointestinal stromal tumors. Endoscopy 2011; 43: $631-634$

[4] Demetri GD, Benjamin R, Blanke C et al. NCCN Task Force report: optimal management of patients with gastrointestinal stromal tumor
(GIST)-expansion and update of NCCN clinical practice guidelines. J Natl Compr Canc Netw 2007; 5: S1-S29

[5] National Comprehensive Cancer Network. Clinical practice guidelines in oncology for soft tissue sarcoma. Version 2 Fort Washington, PA: National Comprehensive Cancer Network; 2009

[6] ESMO/European Sarcoma Network Working Group. Gastrointestinal stromal tumours: ESMO clinical practice guidelines for diagnosis, treatment and follow-up. Ann Oncol 2014; 25: iii21 -iii26

[7] Nishida T, Hirota S, Yanagisawa A et al. Clinical practice guidelines for gastrointestinal stromal tumor (GIST) in Japan: English version. Int J Clin Oncol 2008; 13: $416-430$

[8] Blackstein ME, Blay J-Y, Corless C et al. Gastrointestinal stromal tumours: consensus statement on diagnosis and treatment. Can J Gastroenterol 2006; 20: 157-163

[9] Tanaka J, Oshima T, Hori K et al. Small gastrointestinal stromal tumor of the stomach showing rapid growth and early metastasis to the liver. Dig Endosc 2010; 22: $354-356$

[10] Aso A, Ihara E, Kubo $\mathrm{H}$ et al. Gastric gastrointestinal stromal tumor smaller than $20 \mathrm{~mm}$ with liver metastasis. Clin J Gastroenterol 2013; 6: $29-32$

[11] Boyce GA, Sivak MV, Rösch T et al. Evaluation of submucosal upper gastrointestinal tract lesions by endoscopic ultrasound. Gastrointest Endosc 1991; 37: 449-454

[12] Sepe PS, Moparty B, Pitman MB et al. EUS-guided FNA for the diagnosis of GI stromal cell tumors: sensitivity and cytologic yield. Gastrointest Endosc 2009; 70: 254-261

[13] Hoda KM, Rodriguez SA, Faigel DO. EUS-guided sampling of suspected GI stromal tumors. Gastrointest Endosc 2009; 69: 1218 - 1223

[14] Mohamed A, Yamao K, Sawaki A et al. Diagnostic utility of EUS-guided FNA in patients with gastric submucosal tumors. Gastrointest Endosc 2010; 71: 913-919

[15] Fernández-Esparrach G, Sendino O, Solé M et al. Endoscopic ultrasound-guided fine-needle aspiration and trucut biopsy in the diagnosis of gastric stromal tumors: a randomized crossover study. Endoscopy 2010; 42: 292-299

[16] Sumiyama K, Gostout C], Rajan E et al. Submucosal endoscopy with mucosal flap safety valve. Gastrointest Endosc 2007; 65: 688-694

[17] Kobara H, Mori H, Fujiwara S et al. Bloc biopsy by tunneling method using endoscopic submucosal dissection for an upper gastrointestinal submucosal tumor. Endoscopy 2012; 44: E197-E198

[18] Kobara H, Mori H, Fujihara $\mathrm{S}$ et al. Bloc biopsy by using submucosal endoscopy with a mucosal flap method for gastric subepithelial tumor tissue sampling (with video). Gastrointest Endosc 2013; 77: $141-145$

[19] Matsuzaki I, Miyahara R, Hirooka Y et al. Forward-viewing versus oblique-viewing echoendoscopes in the diagnosis of upper $\mathrm{Gl}$ subepithelial lesions with EUS-guided FNA: a prospective, randomized, crossover study. Gastrointest Endosc 2015; 82: 287 - 295

[20] Fletcher CDM. Clinicopathologic correlations in gastrointestinal stromal tumors. Hum Pathol 2002; 33: 455

[21] Newcombe RG. Improved confidence intervals for the difference between binomial proportions based on paired data. Statist Med 1998; 17: $2635-2650$

[22] Zhang X-C, Li Q-L, Yu Y-F et al. Diagnostic efficacy of endoscopic ultrasound-guided needle sampling for upper gastrointestinal subepithelial lesions: a meta-analysis. Surg Endosc 2016; 30: 2431 - 2441

[23] Inoue T, Okumura F, Mizushima T et al. Assessment of factors affecting the usefulness and diagnostic yield of core biopsy needles with a side hole in endoscopic ultrasound-guided fine-needle aspiration. Gut Liver 2016; 10: 51 - 57

[24] Hikichi T, Irisawa A, Bhutani MS et al. Endoscopic ultrasound-guided fine-needle aspiration of solid pancreatic masses with rapid on-site 
cytological evaluation by endosonographers without attendance of cytopathologists. J Gastroenterol 2009; 44: $322-328$

[25] Hasegawa T, Matsuno Y, Shimoda T et al. Gastrointestinal stromal tumor: consistent CD117 immunostaining for diagnosis, and prognostic classification based on tumor size and MIB-1 grade. Hum Pathol 2002; 33: $669-676$

[26] Kobara H, Mori H, Fujihara S et al. Endoscopically visualized features of gastric submucosal tumors on submucosal endoscopy. Endoscopy 2014; 46: E660-E661
[27] Fujita K, Kobara H, Mori H et al. Differences in miRNA expression profiles between GIST and leiomyoma in human samples acquired by submucosal tunneling biopsy. Endosc Int Open 2015; 3: E665-E671

[28] Khashab MA, Pasricha PJ. Conquering the third space: challenges and opportunities for diagnostic and therapeutic endoscopy. Gastrointest Endosc 2013; 77: $146-148$ 